ARTICLE

Received 11 Apr 2014 | Accepted 21 Jul 2014 | Published 28 Aug $2014 \quad$ DOl: 10.1038/ncomms5764

\title{
Crystal structure and its bearing towards an understanding of key biological functions of EpCAM
}

Miha Pavšič ${ }^{1}$, Gregor Gunčar ${ }^{1}$, Kristina Djinović-Carugo ${ }^{1,2}$ \& Brigita Lenarčič1,3

EpCAM (epithelial cell adhesion molecule), a stem and carcinoma cell marker, is a cell surface protein involved in homotypic cell-cell adhesion via intercellular oligomerization and proliferative signalling via proteolytic cleavage. Despite its use as a diagnostic marker and being a drug target, structural details of this conserved vertebrate-exclusive protein remain unknown. Here we present the crystal structure of a heart-shaped dimer of the extracellular part of human EpCAM. The structure represents a cis-dimer that would form at cell surfaces and may provide the necessary structural foundation for the proposed EpCAM intercellular trans-tetramerization mediated by a membrane-distal region. By combining biochemical, biological and structural data on EpCAM, we show how proteolytic processing at various sites could influence structural integrity, oligomeric state and associated functionality of the molecule. We also describe the epitopes of this therapeutically important protein and explain the antigenicity of its regions.

\footnotetext{
${ }^{1}$ Department of Chemistry and Biochemistry, Faculty of Chemistry and Chemical Technology, University of Ljubljana, Večna pot 113, Ljubljana SI-1000, Slovenia. ${ }^{2}$ Department of Structural and Computational Biology, Max F. Perutz Laboratories, University of Vienna, Campus Vienna Biocenter 5, Vienna AT-1030, Austria. ${ }^{3}$ Department of Biochemistry, Molecular and Structural Biology, Institute Jožef Stefan, Jamova 39, Ljubljana SI-1000, Slovenia. Correspondence and requests for materials should be addressed to M.P. (miha.pavsic@fkkt.uni-lj.si) or to B.L. (email: brigita.lenarcic@fkkt.uni-lj.si).
} 
C ell surface molecules, including cell adhesion molecules, are critically involved in sensing the surroundings of cells. A member of this diverse group of proteins is the epithelial cell adhesion molecule (EpCAM, CD326), a type I transmembrane (TM) glycoprotein of the small GA733 protein family named after the monoclonal antibody raised to the human gastric adenocarcinoma line ${ }^{1}$. In contrast to low expression in normal simple epithelia, EpCAM is frequently expressed at high levels in various epithelial cancers (carcinomas) ${ }^{2}$ and in undifferentiated human embryonic stem cells ${ }^{3,4}$. Elevated EpCAM levels in carcinomas are exploited by several anti-tumor approaches, which deploy EpCAM for drug targeting (reviewed in ref. 5) and a trifunctional bispecific anti-EpCAM anti-CD3 antibody is already approved for use in EU (Catumaxomab) ${ }^{6}$. Several mutations within the EPCAM gene result in truncated or misfolded protein, which is accompanied by incorrect cellular EpCAM localization; these mutations are directly associated with congenital tufting enteropathy (CTE), a rare and severe form of diarrhoea (reviewed in ref. 7).

Initially, EpCAM was postulated to be involved in calciumindependent cell-cell adhesive contacts, which do not resemble the classical junction contacts ${ }^{8-10}$. It was demonstrated that the weak homotypic adhesive function is associated with formation of oligomeric adhesion units in the intercellular space via direct cisand trans-interactions of the larger extracellular parts (EpEX, $27 \mathrm{kDa})^{11,12}$. These units are believed to be anchored to the actin cytoskeleton via interaction of the intracellular tail (EpIC, $3 \mathrm{kDa}$ ) with $\alpha$-actinin ${ }^{13}$. However, it was also demonstrated that EpCAM can have a negative effect on the strength of cell-cell adhesion mediated by E-cadherin thereby promoting cell migration and motility ${ }^{14,15}$. EpCAM also plays an important role in the formation of functional tight junctions and regulation of epithelial integrity via direct interaction with claudin-7 (refs 16-18).

Recently, it was discovered that EpCAM functions as a signalling molecule ${ }^{19}$. Here, the formation in intercellular EpCAM oligomers appears to be a prerequisite for triggering a proliferation-enhancing signalling cascade starting with regulated intramembrane proteolytic cleavage of EpCAM by a membrane protease complex releasing the EpEX and EpIC. The complex of EpIC, FHL2 and $\beta$-catenin is then translocated to the nucleus where it associates with LEF1 and directly affects cell proliferation at transcriptional level ${ }^{19,20}$. However, the outlined aspects of EpCAM biology are far from being well-defined, especially from the structural point of view, since to date no representative structure was available to interpret the experimental observations.

Here, we present the structure of a non-glycosylated mutant form of $\operatorname{EpEX}(\operatorname{EpEX} \Delta)$ at $1.86-\AA$ resolution, a first and exemplary structure of the unique GA733 protein family. In this structure, EpEX $\Delta$ forms a cis-dimer corresponding to a half of the proposed trans-tetrameric intercellular unit. We reveal the cisdimerization interface between the two subunits involving the thyroglobulin type-1A (TY) domain and thereby describe a novel role of the versatile TY protein module. EpCAM cis-dimer could be additionally stabilized by interaction of the TM helices of the two cis-oriented subunits as we explore by molecular dynamics (MD) simulations. We show that the lateral protein surfaces of the $w t$ EpCAM cis-dimer are partially covered with glycan chains as inferred from the position of known $\mathrm{N}$-glycosylation sites. The structure explains the high antigenicity of the surface-exposed small amino-terminal domain that harbours conformational epitopes of the majority of anti-EpCAM antibodies. We provide a model of the trans-tetrameric intercellular unit where the transinteractions involve the glycan-free membrane-distal platformlike surface of $\operatorname{EpEX} \Delta$. Finally, we comment on the design of efficient EpCAM-targeted binder molecules and explain the detrimental effect of CTE-causing mutations on EpCAM structure and function.

\section{Results}

Structure determination. We determined the crystal structure of the human mutant EpEX $\Delta$ in which three Asn-to-Gln mutations were introduced to abolish $N$-linked glycosylation of Asn74, Asn111 and Asn198 residues (Fig. 1a). These mutations enabled us to obtain a highly homogenous protein sample (Supplementary Fig. 1a). After extensive diffraction screening, a complete and highly redundant data set was recorded from a single crystal at $1.54 \AA$ wavelength (Supplementary Fig. 1b). The data were indexed in the $C 2$ space group with unit-cell lengths of $88.0 \times 50.4 \times 67.8 \AA$. Initial phases were calculated using the anomalous signal of intrinsic sulphur atoms, and the structure was solved and refined at $1.86 \AA$ A-resolution (Table 1 ). The overall
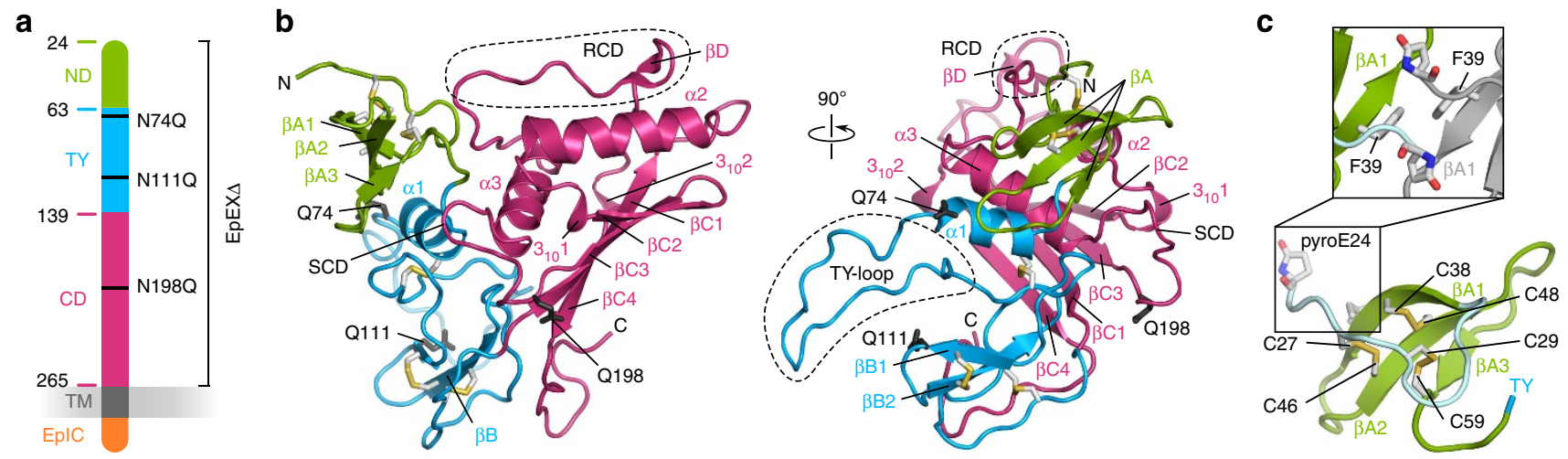

Figure 1 | EpEX $\Delta$ subunit structure. (a) EpEX $\Delta$ is composed of N-domain (ND, green), TY domain (blue) and CD (dark pink). It lacks the TM and intracellular part (EpIC) of full-length EpCAM. In addition, it contains three $\mathrm{N}$-glycosylation-abolishing mutations (indicated by black lines). (b) Cartoon representation of the complete $\operatorname{EpEX} \Delta$ chain contained in the asymmetric unit shown in two different orientations. Color coding is the same as in a. Secondary structure elements are labelled as they appear along the polypeptide chain from $N$ to $C$ terminus (for example, $\beta A$ for $\beta$-sheet $A, \beta A 1$ for first strand in $\beta$ A sheet, $\alpha 1$ and $3_{10} 1$ for first $\alpha$ - and $3_{10}$-helix, respectively). The three glutamine residues ( $N$-glycosylation abolishing mutations) are shown as black sticks. (c) ND shown as cartoon. N-terminal pyroGlu residue (pyroGlu24), Phe39, and all disulphide bridges are shown as sticks. The N-terminal region tethered to the $\beta A$ sheet is coloured in pale cyan. Zoomed-in part shows contact between ND (green) and its copy from the adjacent asymmetric unit (grey). 


\begin{tabular}{|c|c|}
\hline & $\operatorname{EpEX}^{\star} \Delta^{\star}$ \\
\hline \multicolumn{2}{|l|}{ Data collection } \\
\hline Space group & $C 2$ \\
\hline \multicolumn{2}{|l|}{ Cell dimensions } \\
\hline$a, b, c(\AA)$ & $88.02,50.39,67.83$ \\
\hline$\alpha, \beta, \gamma\left({ }^{\circ}\right)$ & $90,128.33,90$ \\
\hline Resolution $(\AA)$ & $27.79-1.86(1.96-1.86)^{\dagger}$ \\
\hline$R_{\text {merge }}$ & $0.055(0.35)$ \\
\hline$I / \sigma(I)$ & $33.98(3.35)$ \\
\hline Completeness (\%) & $99.2(94.3)$ \\
\hline Redundancy & $26.6(8.21)$ \\
\hline \multicolumn{2}{|l|}{ Refinement } \\
\hline Resolution $(\AA)$ & $27.79-1.86$ \\
\hline No. reflections (test set) $)^{\ddagger}$ & $18,528(949)$ \\
\hline$R_{\text {work }} / R_{\text {free }}$ & $0.197 / 0.249$ \\
\hline No. atoms/molecules (per asymmetric unit) & 2,104 \\
\hline Protein & $1,924 / 1$ \\
\hline Water & $147 / 147$ \\
\hline n-Decyl- $\beta$-D-maltopyranoside & $33 / 1$ \\
\hline Mean $B$-factor $\left(\AA^{2}\right)$ & 41.5 \\
\hline Protein & 41.0 \\
\hline Water & 41.8 \\
\hline $\begin{array}{l}\text { n-Decyl- } \beta \text {-D-maltopyranoside } \\
\text { r.m.s. deviations }\end{array}$ & 44.9 \\
\hline Bond lengths $(\AA)$ & 0.010 \\
\hline Bond angles $\left({ }^{\circ}\right)$ & 1.273 \\
\hline
\end{tabular}

${ }^{*}$ One crystal was used for data collection and refinement. Data was collected at a wavelength of $1.542 \AA$ on a Bruker AXS Microstar rotating-anode X-ray diffractometer.

-Values in parenthesis are for the highest-resolution shell.

$\ddagger$ Number of reflections corresponds to merged data set used in refinement.

quality of the electron density map was high except for some surface regions, which display a higher B-factor (Supplementary Fig. 1c). An extended blob of electron density that could not be assigned to the EpEX $\Delta$ polypeptide chain was modelled as a crystallization additive $n$-decyl- $\beta$-D-maltopyranoside, bound in a narrow hydrophobic pocket (Supplementary Fig. 2).

Overall subunit structure of human EpEX $\Delta$. The polypeptide chain of EpEX $\Delta$ is folded into three domains (ND, TY and CD, see Fig. 1a) arranged in a triangular fashion where each domain contacts the other two (Fig. 1b). The first residue immediately after the signal peptide cleavage site $(\mathrm{Gln} 24)$ is modified to pyroglutamate (pyroGlu), which is a common modification of an extracellular N-terminal and has already been observed in recombinant $\mathrm{EpEX}^{21}$ (Fig. 1c). The small and compact disulphide-rich N-terminal domain of 39 amino-acid residues (N-domain or ND, pyroGlu24-Leu62; green in Fig. 1) lacks an extensive hydrophobic core. Instead, its structure is stabilized by three closely spaced disulphide bridges (Cys27-Cys46, Cys29Cys59 and Cys38-Cys48), which tether the N-terminal region of the chain (pyroGlu24-Leu34) to the concave face of a curved three-stranded $\beta$ sheet with strand order 1-2-3 ( $\beta$ A, Fig. 1c). The disulphide bond pattern observed in our structure confirms the previously determined cysteine bonding ${ }^{21}$. A similar triple disulphide bond pattern is found in other small protein domains, for example in the Cripto CFC domain ${ }^{22}$. According to the alignment of 3D structures in the PDB database $\mathrm{P}^{23}$, the ND domain is most similar to the WW protein module ${ }^{24}$. However, there are considerable differences: (i) ND lacks the two highly conserved tryptophan residues found in WW modules, and (ii) WW modules do not contain disulphide bridges and lack the $\mathrm{N}$-terminal region present in ND. The solvent-exposed regions of the ND are negatively charged (N-terminal region) or polar (convex side of the $\beta \mathrm{A}$ sheet) with an exception of Phe39 within the FVNNN sequence motif (conserved in primates; Supplementary Fig. 3a). In our crystal structure, the side chains of two Phe39 residues from adjacent asymmetric units are involved in parallel-displaced $\pi-\pi$ interactions (inset in Fig. 1c).

ND is followed by the TY domain (Ala63-Arg138; blue in Fig. 1), which is a TY module with a characteristic fold stabilized by an evolutionarily conserved disulphide bridge pattern (Cys66Cys99, Cys110-Cys116 and Cys118-Cys135 as numbered in EpCAM $)^{21}$ and a CWCV sequence motif within the $\beta B$ ribbon ${ }^{25}$. The secondary structure elements ( $\alpha 1$ helix and $\beta B$ ribbon) and neighbouring regions of the polypeptide chain form a small hydrophobic core additionally stabilized by the three disulphide bridges. The region between the $\alpha 1$ helix and the $\beta B$ ribbon of the TY domain protrudes as a loop (TY loop) from the otherwise compact EpEX $\Delta$ molecule (Fig. 1b).

The third domain (carboxy-terminal domain, C-domain or CD, Val139-Lys265; dark pink in Fig. 1) belongs to the $\alpha+\beta$ fold class. The helices are clustered on one side of the slightly twisted $\beta C$ sheet, which forms a negatively charged polar concave surface on the side of the EpEX $\Delta$ molecule (Fig. 1b). Here, the two amphipathic $\alpha$ helices are on the hydrophobic convex side within the regions connecting the $\beta$ strands of the $\beta C$ sheet. This fold resembles the fold of some unrelated protein domains, for example, the RNA binding domain of Bacillus subtilis $\mathrm{YxiN}^{26}$ and the mature ectodomain of human receptor-type protein tyrosine phosphatase IA-2 (ref. 27). The major differences are in the loop regions connecting the secondary structure elements where in EpEX $\Delta$ the most prominent part is the region connecting the $\alpha 3$ helix with the $\beta C$ sheet (Lys221-Thr247). The central part of this region (His227-Gln239, 'ridge on CD' or RCD) contains a stretch of polypeptide chain that runs in parallel to the $\alpha 2$ helix and a short $\beta$ hairpin. In addition, two residue triplets (Pro178-Phe180 and Pro244-Gln246) within CD have a $31_{10}$ conformation resulting in two interlocked tight turns on each side of the $\beta C$ sheet ( $3_{10} 1$ and $3_{10} 2$ in Fig. 1b). Region Lys202-Asn205 ('side loop on CD' or SCD) does not have a well-defined electron density indicating some flexibility in this surface-exposed turn (Supplementary Fig. 1c).

The EpEX cis-dimer. In vivo, EpCAM forms a cis-dimer where the two subunits laterally interact on the cell surface ${ }^{11,12}$. Indeed, EpEX $\Delta$ crystallized as a dimer, which is formed by two EpEX $\Delta$ chains from adjacent asymmetric units related by the crystallographic twofold symmetry axis (Fig. 2a,c). The dimeric form is also present in solution as demonstrated by crosslinking (Fig. 3a) and size-exclusion chromatography ${ }^{28}$. The extensive buried surface area of $1,980 \AA^{2}$ (per subunit) with a solvation free energy gain on formation of the interface of $-8.6 \mathrm{kcal} \mathrm{mol}^{-1}$ and a $P$ value of 0.358 as calculated by PISA $^{29}$ indicates strong and specific interaction. The interaction surface represents $14 \%$ of the subunit surface area; the upward-oriented C-terminal region Met261-His266 was excluded from this calculation (see below). The most prominent interaction region is formed by the TY loop of one subunit and a concave $\beta C$ sheet of the other subunit (yellow in Fig. 2c). Specifically, Arg80, Arg81 and Lys83 of the TY loop of one subunit are involved in electrostatic interactions with the acidic patch formed by Glu147, Glu187 and Asp194 of the $\beta C$ sheet of the other subunit (Fig. 2b). The side chain of Gln74, which in $w t$ EpCAM corresponds to glycosylated Asn74, is oriented towards the solvent and is not involved in the dimerization interactions.

The plausible orientation of EpEX $\Delta$ (as a part of the full-length EpCAM) relative to the cell membrane can be inferred by the 
position of $\mathrm{N}$ and $\mathrm{C}$ termini. In full-length EpCAM, the $\mathrm{C}$ terminus of the extracellular part is connected to the TM region, suggesting that this is the membrane-proximal part. Since both $\mathrm{C}$ termini in the $\operatorname{EpEX} \Delta$ structure are on the same side of the dimer (side orientation in Fig. 2a), we conclude that this dimer architecture represents extracellular parts of two EpCAM molecules on the surface of the same cell, and will refer to it as a cis-dimer. The membrane-proximal part encompasses the TY domain and the $\beta C$ sheet of the C-domain (Fig. 2a) and forms a tip of the cis-dimer with a net negative charge (Glu85, Asp128 and 130, Glu132; Fig. 2b) suggesting that it is not in contact with the central hydrophobic part of the lipid bilayer as part of the fulllength membrane-embedded EpCAM. However, the C-terminal part of the EpEXA chain (from Met261 to Lys265 plus the first histidine residue His266 of the $\mathrm{His}_{6}$-tag) is curved upwards (Fig. 2a) and forms several polar contacts with parts of TY domains from both subunits (Supplementary Fig. 4). In $w t$ glycosylated EpEX such conformation could be sterically hindered by the glycan chain attached to the Asn111 within the $\beta \mathrm{B}$ of TY (Fig. 2c). In full-length $w t$ EpCAM, this region is probably oriented 'downwards' as discussed in the section regarding the TM helix (below). The membrane-distal part of this cis-dimer forms a central groove edged by the two RCDs and diagonally protruding small $\mathrm{N}$-domains (Fig. 2a,c). The inner surface of the groove is formed by diametrically opposing basic and acidic patches (plus and minus sign labels in Fig. 2b).

The EpEX $\Delta$ cis-dimer has the shape of a heart (view along the $\mathrm{RCD}$ ) or trapezoid (view perpendicularly to RCD) with a narrowed membrane-proximal part (Fig. 2c). Such dimer would protrude $\sim 5 \mathrm{~nm}$ from the cell surface. In humans, EpCAM is glycosylated on the three $N$-glycosylation sites: Asn74 at the start of the TY loop, Asn111 within $\beta B$ of the TY, and Asn198 between $\beta C$ and $\alpha 3$ within $C^{30}$. The glycosylation is heterogeneous and at least one site is occupied by a high-mannose chain ${ }^{31}$. The EpEX $\Delta$ structure with modelled high-mannose chains attached at all three sites shows that the glycans partially cover the sides of the membrane-proximal part (Asn111 and Asn198) or are located at the start/end of the central groove of membrane-distal part of the cis-dimer (Asn74; Fig. 2c). The top membrane-distal part of the molecule formed by RCD and ND is free of glycan chains.

Cleavage within TY disrupts the cis-dimer. In vivo, full-length glycosylated EpCAM $(\sim 38 \mathrm{kDa})$ is proteolytically processed within a protease-sensitive region containing the dibasic site Arg80-Arg81 of the TY loop ${ }^{32-34}$. The resulting $6 \mathrm{kDa}$ N-terminal and $32 \mathrm{kDa}$ C-terminal fragments remain connected by a disulphide bond. In $\operatorname{EpEX} \Delta$, the larger C-terminal fragment would correspond to a $28 \mathrm{kDa}$ fragment lacking the TM and the cytosolic part. To resolve the impact of this cleavage on structural integrity of EpCAM, we generated the cleaved form of EpEX $\Delta$ and analysed its dimerization potential by chemical crosslinking. The cleaved form was generated by treating the purified EpEX $\Delta$ ( $\sim 33 \mathrm{kDa}$, lanes 1 and 5 in Fig. 3a) with human cathepsin L. The major cleavage site was at Gly79-Arg80 with a minor cleavage also at Leu78-Gly79, that is, both within the same proteasesensitive region as reported previously using several other proteases $^{34}$. In the absence of crosslinker and under nonreducing conditions, the 6 and $28 \mathrm{kDa}$ fragments remain connected via a disulphide bond (lane 6 in Fig. 3a). However, under reducing conditions, the $6 \mathrm{kDa}$ fragment is released from the rest of the molecule $(28 \mathrm{kDa}$, lane 2 of Fig. $3 \mathrm{a})$. A band the mass corresponding to a EpEX $\Delta$ dimer $(\sim 66 \mathrm{kDa})$ was observed only with crosslinked intact EpEX $\Delta$ (lanes 3 and 7 in Fig. 3a). Although part of cleaved and crosslinked EpEX $\Delta$ has under reducing conditions (lane 4) similar electrophoretic mobility than a
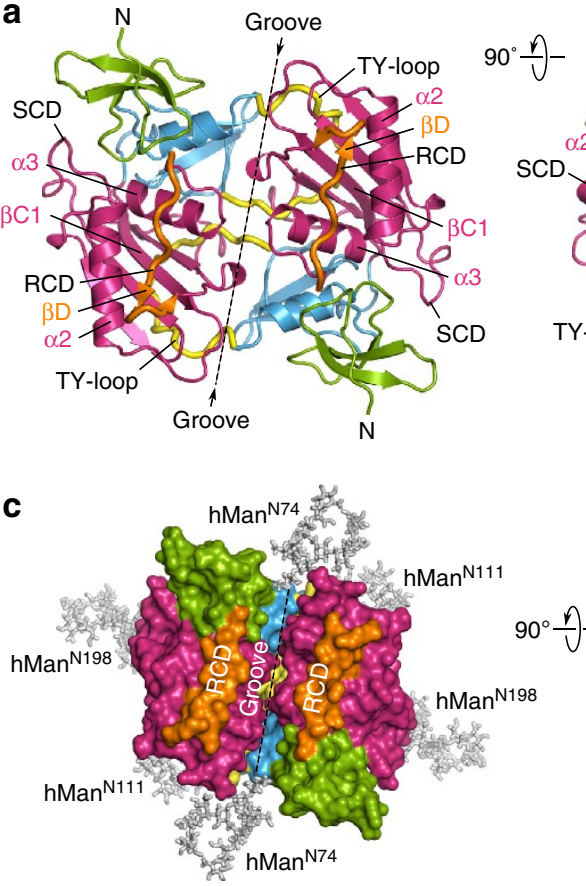

Membrane-distal

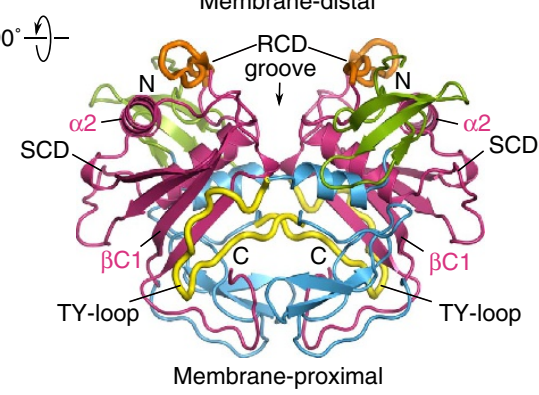

Membrane-proximal b Membrane-distal

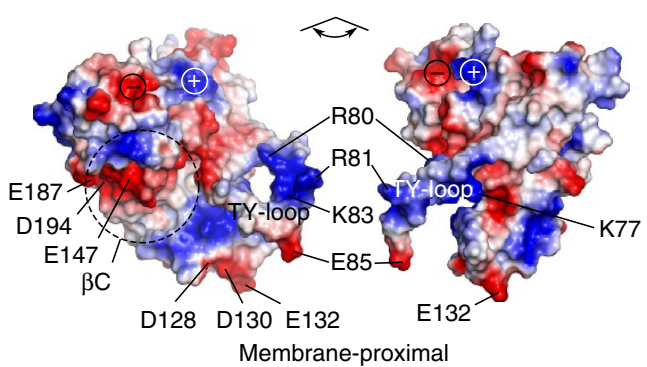

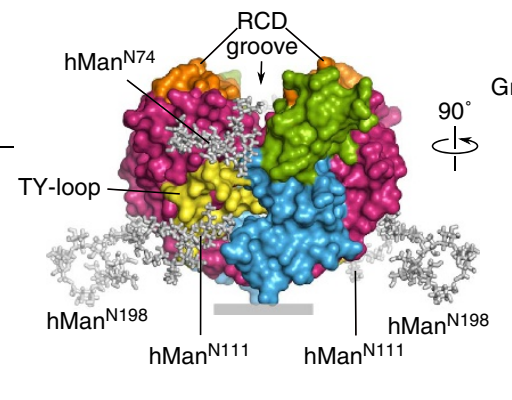

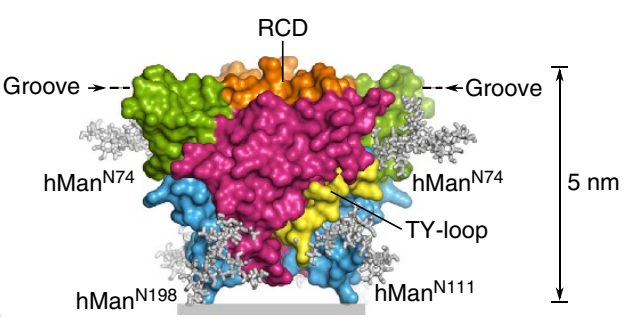

$\mathrm{hMan}^{\mathrm{N} 198}$

Figure 2 | Structure of EpEX $\mathbf{\Delta}$ cis-dimer. (a) Cartoon representation of EpEX $\Delta$ cis-dimer in two different orientations (top, side). (b) The molecular surface of EpEX $\Delta$ cis-dimer, color coded by electrostatic potential. Subunits are moved apart and rotated to reveal the oppositely charged interaction regions. (c) Molecular surface of EpEX $\Delta$ cis-dimer with modelled high-mannose chains at N-glycosylation sites of wt EpCAM (Asn74, Asn111 and Asn198) shown in three different orientations relatively to the membrane (grey bar). The three domains of each subunit in a,c are color coded as in Fig. 1.

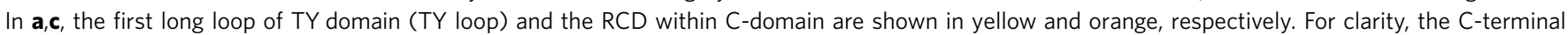
region of EpEXA (from Met261 to Lys265 plus His266) is not shown in b,c. 

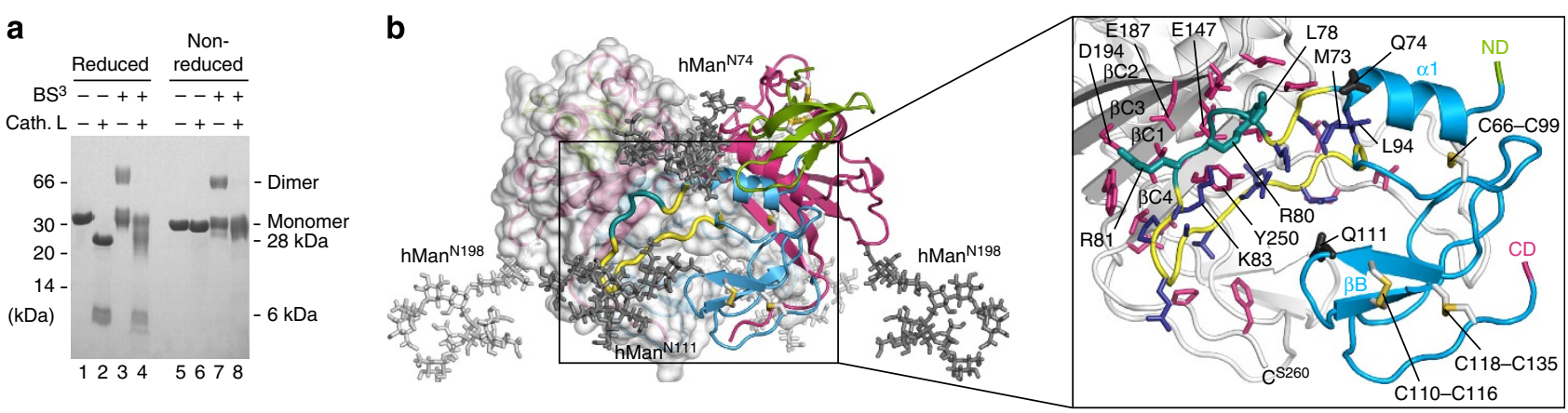

Figure 3 | Role of TY loop in cis-dimerization. (a) SDS-PAGE analysis of crosslinked intact and cathepsin L-cleaved EpEX $\Delta$ samples under reducing and non-reducing conditions. Cleavage with cathepsin $L$ results in 6 and $28 \mathrm{kDa}$ fragments connected by a disulphide bond. Only intact EpEX $\Delta$ forms a stable dimer, which can be crosslinked by $\mathrm{BS}^{3}$. (b) Overall view (bottom) of the model of glycosylated EpEX cis-dimer shows that the access to the protease-sensitive Leu78-Gly79-Arg80-Arg81 (dark teal ribbon) within the TY domain between is partially obscured by glycan residues (grey sticks). TY loop and ND, TY and CD domains are color coded as in Fig. 2. Detailed view (top) of TY-loop residues (dark purple) interacting with residues of $\beta C$ sheet (grey, side chains within $4 \AA$ of TY loop shown in dark pink). The Arg80 and Arg81 (dark teal sticks) interact with Asp194, Glu154 and Glu147. Gln74 and GIn111 (black sticks) introduced by glycosylation-abolishing mutations within TY correspond to glycosylated Asn residues in wt EpEX. The three disulphide bonds of TY are shown as sticks. Under non-reducing conditions, the 6 and $28 \mathrm{kDa}$ fragments (ND plus $\alpha 1$ and the rest of TY plus CD, respectively) remain connected by the Cys66-Cys99 bond. For clarity, the C-terminal region of EpEX $\Delta$ (from Met261 to Lys265 plus His266) is not shown.

non-cleaved EpEX $\Delta$ such molecule cannot form a dimer anymore (lanes 4 and 8 in Fig. 3a). Cleavage using glycosylated EpEX as a substrate resulted in the same fragment pattern as for the EpEX $\Delta$ (Supplementary Fig. 5). Also, the same fragment pattern could be generated using cathepsin $\mathrm{K}$, which also cleaves at the Gly79-Arg80 site.

In the structure of the EpEX $\Delta$ cis-dimer, the side chain of Leu78 is oriented towards the solvent while the basic side chains of Arg80 and Arg81 of one subunit are oriented towards the $\beta C$ sheet of the CD of the other subunit where they are involved in cis-dimer-stabilizing interactions with acidic (Arg80 with Glu147, Arg81 with Asp194) and other residues (Val190, Gln246 and Leu248; Fig. 3b). In the EpEX cis-dimer, access to the cleavage site Leu78-Gly79-Arg80 could be partially hindered by glycan chains attached to Asn74 (at the beginning of TY loop) and Asn111 (within turn in $\beta B$ ) as inferred from the model of glycosylated EpEX (Fig. 3b).

Role of TM helix in cis-dimerization. In the crystal structure of the EpEX $\Delta$ cis-dimer, the $\mathrm{C}$ termini are curved upwards (Fig. 2a). However, in full-length, membrane-anchored EpCAM, the C-terminal region of the extracellular part must be oriented towards the membrane to be connected to the TM part. Considering that the cell surface full-length EpCAM is a cis-dimer as inferred from the crystal structure and from the crosslinking experiments $^{12}$, such downward orientation would bring the C-terminal regions of the two subunits into close proximity. Therefore, it is plausible to assume that also the TM part (single helix per chain) forms a dimer with potential to additionally stabilize the EpCAM cis-dimer. To investigate the dimerization propensity of the EpCAM TM helix, we performed coarsegrained (CG) MD simulations of two modelled EpCAM TM helices embedded in a lipid bilayer. The two EpCAM TM helices were allowed to diffuse freely within the bilayer patch from their initial positions separated by $50 \AA$.

The plot of interhelical distance versus time in each of the simulations shows that once the two helices encountered each other the helix dimer stayed stable throughout the simulation (runs 1 and 3-6 in Fig. 4a). In one of the simulation runs, the two helices did not encounter each other (run 2 in Fig. 4a). In this run, the interhelical crossing angle distribution is flattened and centred near zero degrees as compared with two sharp peaks of bimodal interhelical crossing angle distribution observed in the other runs (Fig. 4b). Here, the two peaks of the crossing angle distribution are at $-30^{\circ}$ and $+30^{\circ}$, corresponding to right- and left-handed packing. Higher frequencies of the $-30^{\circ}$ peak indicate that a right-handed packing is favored. Preference for certain crossing orientation has already been observed in CG MD simulations of the $w t$ glycophorin A TM helix dimer; in contrast to this, the glycophorin A TM dimer disruptive mutants showed a more uniform distribution between the right- and left-handed crossing angle ${ }^{35}$. In a representative CG model of a right-handed EpCAM TM helix dimer, the two helices are arranged almost symmetrically and the crossing region encompasses the residues Val276-Val280 (Fig. 4c). Here, the Ile277 side chain of one helix locks into a pocket formed by side chains of Val276, Val279 and Val280 from the other helix. On the basis of these results, we predict that the EpCAM TM helix has a propensity to form a dimer when embedded in a lipid bilayer.

Model of EpCAM intercellular unit. It is believed that EpCAMmediated adhesion and signalling involves formation of intercellular trans-oligomers. We used docking to generate a model of a trans-tetrameric intercellular unit based on our crystal structure of the cis-dimer together with available experimental data: (i) the $\mathrm{ND}$ is not required for intercellular adhesion ${ }^{12}$, (ii) juxtaposed cell membranes at EpCAM adhesion sites are separated by $10-14 \mathrm{~nm}^{10}$ and (iii) lack of EpCAM $N$-glycosylation has no influence on its adhesive properties ${ }^{12}$. Our model (Fig. 5) satisfies the outlined requirements, also in terms of intermembrane separation where the extended C-terminal region contributes an additional $1 \mathrm{~nm}$ separation to the $5 \mathrm{~nm}$ membrane-to-tip distance of the EpEX $\Delta$ cis-dimer (Fig. 2c). The trans-interaction surface is formed by the membrane-distal parts of the EpEX $\Delta$ dimer including the RCD while the glycan residues project sideways from the rhombohedral-shaped trans-tetramer (Fig. 5).

\section{Discussion}

The presented EpEX $\Delta$ structure reveals that the extracellular part of EpCAM is folded in a compact shape and is not extending from the cell surface in a linear way as portrayed in early models. In the crystal structure, EpEX $\Delta$ is present as a dimer and on the basis of a large interaction surface and orientation of the $\mathrm{C}$ termini, we postulate that this form represents a cis-dimeric cell surface form 
of EpCAM. The dimerization crucially depends on the TY domain, which adds dimerization to the functional repertoire of this versatile protein module besides cysteine protease inhibition as in p41 invariant chain ${ }^{36}$, inhibition and substrate behaviour as in testican-1 (ref. 37), and binding of insulin-like growth factor 1 as in IGF binding proteins ${ }^{38}$. The first loop of the TY module is the most divergent region of TY domains from various protein architectures and represents one of the functional determinants of these modules ${ }^{39}$. The TY domain of EpCAM does not inhibit cysteine cathepsins (M.P. and B.L., unpublished data); one of the more obvious reasons are the steric hindrances imposed by the
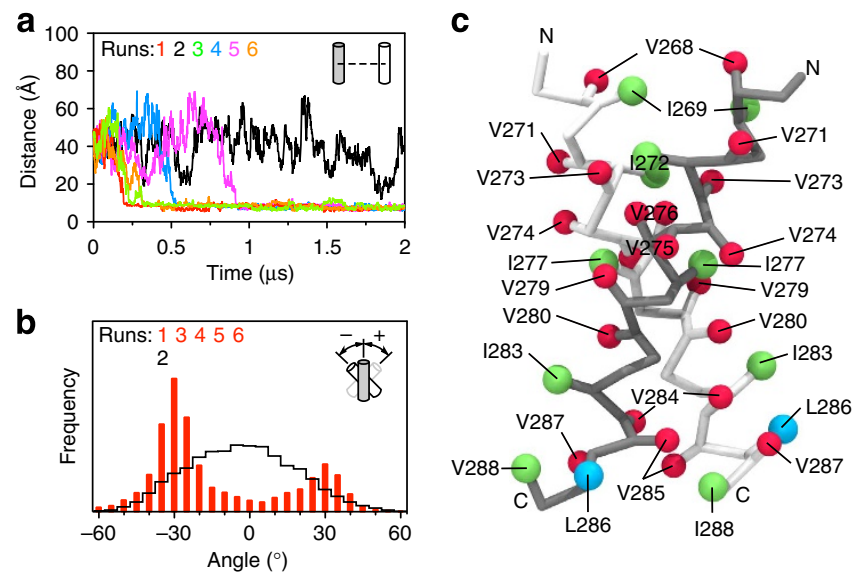

Figure 4 | TM helix dimerization contributes to stability of EPCAM cisdimer. (a) Interhelical distance calculated from six CG MD simulations started from the same initial state. (b) Interhelical crossing angle distribution in combined simulation runs 1 and 3-6 (red), and run 2 (black). For combined runs only those parts where the two helices formed a dimer were used for calculation. (c) Representative CG dimer model TM helices of EpCAM calculated by MD simulation. Backbones of the two helices are shown as light and dark gray sticks. CG side chains of leucine, isoleucine and valine residues are shown as blue, green and red spheres, respectively. Labels follow residue numbering in full-length EpCAM. long first loop that might prevent its binding to the active site cleft of the protease. Instead, this first loop (TY loop) is directly involved in interactions with the $\mathrm{CD}$ of the other subunit in the same dimer. This cis-dimer could be additionally stabilized by extension of the dimerization interface to the TM part with implications in intramembrane interactions of EpCAM with other proteins and vice versa. Moreover, the intramembrane dimerization potential of EpCAM TM parts could be influenced by the composition of the lipid bilayer. In our MD simulations, we used the simple 1-palmitoyl-2-oleoylphosphatidylcholine lipid bilayer; however, it has been reported that a complex of EpCAM and claudin-7 is recruited to the complex glycolipidenriched tetraspanin microdomains ${ }^{40}$, which are known modifiers of adhesion and signalling functions of TM molecules.

The key role of TY loop in dimerization interactions is also illustrated by the TY loop-cleaved form of EpEX $\Delta$, which is not able to form a stable cis-dimer anymore. However, the cleavage site does not seem to be easily accessible to proteases since it is directly involved in cis-dimerization interactions. Even more, in glycosylated EpEX (model in Fig. 3b) the access to the cleavage site could be sterically hindered by the glycan chains attached to Asn74 and Asn111 (both are glycosylated in insect cells ${ }^{21}$ ); however, this EpEX form is cleaved almost to the same extent as the non-glycosylated EpEXA (Supplementary Fig. 5). This suggests that proteases act on monomeric EpEX $\Delta$ or on a loosened cis-dimer where the cleavage site within the TY loop would be more accessible. Translated to the cell surface EpCAM, this would mean that there is a dynamic equilibrium between monomeric and dimeric EpCAM as already indicated ${ }^{11}$. Another possibility is that the TM helix dimer stays intact and that the extracellular parts temporarily move apart to a certain degree giving protease opportunity to access the identified cleavage site. The monomer-dimer equilibrium could be affected by various factors, such as lipid composition or association with other proteins. Also, the EpEX dimer could be destabilized by a $\mathrm{pH}$ drop, which we already demonstrated in vitro ${ }^{28}$. A locally acidic environment with $\mathrm{pH}$ even below 6.5 (ref. 41) is often found in tumours and could at the same time maintain the activity of various proteolytic enzymes released into the extracellular space.

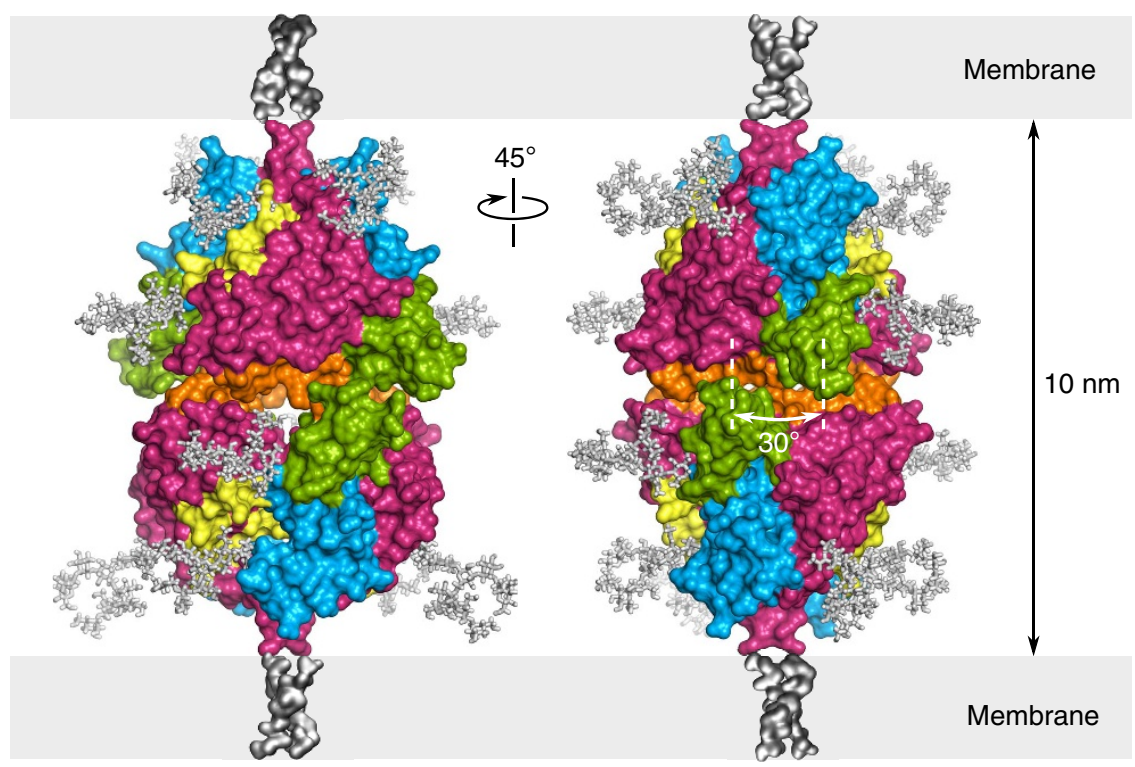

Figure 5 | Model of a trans-tetrameric intercellular unit. In the most probable relative orientation, the angle between the two cis-dimers is $30^{\circ}$. The model is shown in two different orientations with modelled high-mannose glycan chains (as in Fig. 2c) and dimers of the TM parts (as in Fig. 4b). The $\mathrm{C}$ termini were modelled in an extended conformation. EpEX domains and regions (TY loop and RCD) are color coded as in Fig. 2. 
Even more, while EpCAM is not expected to be a physiological substrate of trypsin and other proteases used in previous studies, the lysosomal cysteine cathepsins used in our study are under some conditions released to the extracellular space where they actively participate in ECM remodelling or even ECM degradation, for example in cancer (reviewed in ref. 42). Therefore, it is tempting to speculate that they are involved in in vivo EpCAM cleavage affecting the oligomeric state, associated functions and turnover of EpCAM at the cell surface. The slight differences in observed cleavage site (Gly79-Arg80 as observed with cathepsins L and $\mathrm{K}$, and $\operatorname{Arg} 80-\operatorname{Arg} 81$ as reported previously ${ }^{33}$ ) could be a result of further N-terminal trimming at Gly79 or action of different proteases at this protease-sensitive region in different cells.

In humans, EpCAM is heterogeneously glycosylated at the three $N$-glycosylation sites ${ }^{43}$ and it has been demonstrated that glycosylation at Asn198 is crucial for stability ${ }^{30}$. As inferred from the model of glycosylated EpEX (Fig. 2c), this glycosylation site is located close to the membrane where it might influence trafficking or interactions with proteins affecting EpCAM turnover rate. The arrangement of glycan chains attached to Asn111 and Asn198 in the cis-dimer resembles the tripod-like arrangement of glycans in the membrane-proximal region of ICAM-2 where they are assumed to play a role in a proper orientation of ICAM-2 with regard to the cell surface and interacting cells ${ }^{44}$. This is also in line with our model of the intercellular EpCAM trans-tetramer (Fig. 5). This model suggests sporadic intercellular contacts unlike the extensive contacts based on a zipper-like model of the C-cadherin junctions ${ }^{45}$. Comparison of the interaction surface between two subunits in the EpEX $\Delta$ cis-dimer $\left(2,438 \AA^{2}\right)$ and between two cis-dimers in our model of the trans-tetramer $\left(800 \AA^{2}\right)$ is also in line with the determined dissociation constants $(\sim 10 \mathrm{nM}$ and $\sim 10 \mu \mathrm{M}$ for dimer-to-monomer and tetramer-to-dimer, respectively) ${ }^{11}$. The high dissociation constant for the tetramer-to-dimer equilibrium is in compliance with the absence of tetramer in crosslinking experiments (Fig. 3a). In cells, the tetramer-to-dimer equilibrium could be strongly affected by the crowding effect in the intercellular space along with the restricted degrees of freedom due to membrane anchoring. However, in case of conformational changes in the RCD a different relative orientation of the two dimeric subunits could be favored. Also, the conformation of RCD in our structure could be imposed by the bound n-decyl- $\beta$-D-maltopyranoside (Supplementary Fig. 2).

The amino-acid sequence of EpEX is highly conserved in organisms like primates, ungulates and rodents, and to a certain extent even in other groups of organisms such as fishes, amphibians and reptilians (Supplementary Fig. 3a). Specifically, the most conserved parts are the core of the TY ( $\alpha 1$ helix and the region including the $\beta \mathrm{B}$ ) and the secondary structure elements of the $\mathrm{CD}$ (except the $\alpha 2$ helix). Also, in organisms ranging from primates through ungulates and rodents to birds, reptiles and amphibians the TY-loop region is largely conserved including the dibasic site (Arg80-Arg81 in humans). Since the $\beta C$ and the TY loop are critically involved in cis-dimerization, it is reasonable to assume that also in all these organisms EpEX also forms a cisdimer. Interestingly, fishes have a four to six amino-acid residue insertion in this region indicating a somewhat different cisdimerization mechanism if even applicable. Sequence conservation mapped to the structure of EpEX cis-dimer revealed that most differences lie in the membrane-distal part of the dimer (Supplementary Fig. 3b). This region, which is formed by the ND and the $\alpha 2$ helix plus RCD of the $\mathrm{CD}$, may represent the environment-sensing part of EpCAM while the lateral parts may be involved in interactions with other (trans)membrane proteins on the surface of the same cell. In the light of this information, the
RCD and possibly the nearby $\alpha 2$ are proposed to be directly involved in EpCAM trans-tetramerization as described in our paper.

An important aspect of EpCAM biology is the proteolytic release of EpIC, which is implicated in proliferative signalling. Since the cleavage of cell surface EpCAM could be triggered by soluble EpEX added to the cell culture, it was suggested that formation of intercellular oligomeric units is a prerequisite or a trigger for the initial cleavage event ${ }^{19}$. The formation of a transtetramer could be accompanied by conformational changes, which are translated to the C-terminal region of the extracellular part close to the membrane resulting in cleavage-promoting structural changes. To date, several cleavage sites were identified. For example, it has been reported that in bladder cancer patients, EpEX is shed via cleavage at the Ala266-Gly267 site ${ }^{46}$ corresponding to the beginning of the EpCAM TM part. This cleavage would directly release the EpEX from the cell surface since it would break the membrane anchoring provided by the TM region. In mouse, other cleavage sites were observed, which in human EpCAM correspond to Ser228-Lys229 ( $\alpha$-site, close to the $\beta D$ within the RCD, Fig. 1b), Tyr250-Tyr251 ( $\beta$-site, within the $\beta C 4$, Fig. $3 \mathrm{~b}$ ), and several sites within the TM region including the $\gamma_{2^{-}}$and $\gamma_{1}$-sites Ala270-Val271 and Val273-Val274, respectively (Fig. $4 \mathrm{c})^{47}$. The $\alpha$-cleavage site within the RCD would imply that the protease active site is at least $5 \mathrm{~nm}$ from the cell surface as inferred from the EpEX dimensions or is a result of proteolytic action of a protease at the surface of a neighbouring cell or even a soluble protease. While the $\alpha$-site appears accessible in the cis-dimer, the $\beta$-cleavage within $\beta \mathrm{C}$ suggests a dynamic equilibrium between EPCAM monomer and cis-dimer (discussed above) since in the cis-dimer this cleavage site is inaccessible. It is tempting to speculate that the $\beta$-cleavage is triggered by a local drop of $\mathrm{pH}$ since this both destabilizes the EpEX cis-dimer and at the same time enhances the activity of the BACE1 protease involved in the cleavage ${ }^{47,48}$. However, both cleavages within the $\mathrm{CD}$ as identified using murine EpCAM seem to be incompatible with the model where the formation of trans-intercellular contacts triggers the signalling-associated cleavage. The intramembrane cleavages, particularly at both $\gamma$-sites, could interfere with TM helix dimerization since this region is located at the crossing region of the TM helix dimer as predicted by MD simulations (Fig. 4c). However, the $\gamma$-cleavages are preceded by the cleavages within the extracellular part therefore we speculate that the cleavage-induced destabilization of the extracellular region conformationally and/or sterically paves the way for $\gamma$-cleavages within the TM part. Recently, additional cleavage sites at the extracellular part were identified: a cleavage at the mouth of the central groove in cis-dimer within a region connecting the $\beta \mathrm{Cl}$ to the $\alpha 2$ helix of the $C D$, and a several cleavages within and near the SCD (Figs $1 \mathrm{~b}$ and $2 \mathrm{a})^{49}$. The role of all these cleavages and the exact mechanism by which they occur remains to be elucidated.

The structure of EpEX $\Delta$ also addresses the effect of CTEcausing mutations on EpCAM structural integrity. Most of the CTE-associated mutations within the EPCAM gene result in truncated forms of EpCAM lacking most of the $\mathrm{CD}$ along with TM and EpIC (reviewed in ref. 7), and only two result in an EpCAM molecule impaired in some other way-lacking either the Trp143-Thr164 region (corresponding to $\alpha 2$ helix and one of the two middle strands in $\beta C$ sheet; Fig. 1b) or having the Cys66 within TY mutated to $\mathrm{Tyr}^{50}$. The deletion most probably significantly impairs EpCAM folding by linking together two residues, which are more than $20 \AA$ apart in the wt EpCAM. The second mutation results in the absence of a stabilizing disulphide bond between Cys66 and Cys99 (Fig. 3b) and also introduces a residue with a bulkier side chain seriously perturbing the core of the TY. This latter mutation could impair formation of EpCAM 


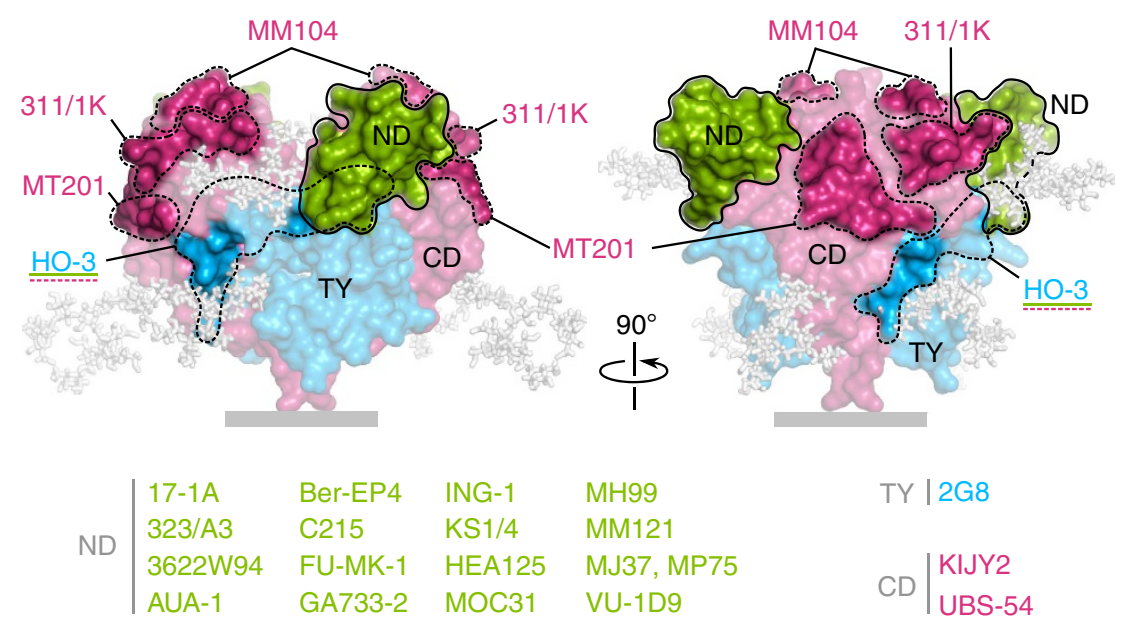

Figure 6 | Epitopes of anti-EpEX antibodies mapped to a pale-coloured surface model of glycosylated EpEX cis-dimer. The identified epitope regions and the most antigenic $\mathrm{N}$-domain are depicted as intensively shaded surface regions. Listed below are other anti-EpEX antibodies for which the exact epitope is unknown. Antibody names are color-coded by the domains they recognize (ND, TY and CD in green, blue and dark pink, respectively). Epitopes of antibodies HO-3, 17-1A and MT201 are targeted by Catumaxomab, Edrecolomab and Adecatumumab, respectively.

cis-dimers or even prevent normal folding of EpCAM and adoption of the compact overall structure.

Another disease-associated aspect is the use of EpCAM as prognostic tool and for targeted drug delivery. The exceptional antigenicity of the ND has been recognized some time ago ${ }^{34}$ and is in line with the location of this domain at a very exposed position in the EpEX $\Delta$ cis-dimer (Figs 2a,c and 6). Moreover, the majority of epitopes mapped to this domain are destroyed by reduction of disulphide bonds confirming their conformational nature dependent on correct pattern of disulphide bonds within the compact ND. Other known polypeptide epitopes are primarily located in the membrane-distal or upper lateral part of the EpEXA cis-dimer, which is again in line with their higher accessibility due to lack of glycan chains and greater cell membrane-epitope distance (Fig. 6). In addition, the membraneproximal surface regions could be masked by other (interacting) membrane proteins. Still, while the ND is targeted by a plethora of antibodies, some surface-exposed regions of the TY and CD domain remain unexplored. Also, dissociation of the EpCAM dimer as speculated above would reveal additional potential epitopes previously located within buried surface and therefore inaccessible. Interestingly, the epitope of the HO-3 antibody, which is the EpCAM-binding part of the EU-approved Catumaxomab $^{6}$ extends from the part of ND through TY loop to part of the CD. This suggests that the HO-3 antibody/ Catumaxomab recognizes EpCAM in a cis-dimeric form. At the same time, the epitope size indicates that antibody regions adjacent to the complementarity determining regions are involved in EpCAM recognition. In our model, the epitope of HO-3 is partially covered with glycan chains, which are, however, flexible and could move from the mentioned areas thereby exposing the polypeptide epitope (Fig. 6).

In recent years, the EPCAM paralogue Trop2, the only other member of the GA733 family, is gaining attention as a stem ${ }^{51}$ and carcinoma cell marker ${ }^{52}$. The two proteins share a $49 \%$ sequence identity and for Trop2 a signalling function via regulated intramembrane proteolysis reminiscent of EpCAM was demonstrated $^{53}$. However, no adhesive function has been reported to date. It is important that the similarities/differences are taken into account when developing diagnostic/prognostic and therapeutic approaches and great care is needed when analysing the results with regard to both EpCAM and Trop2.

\section{Methods}

Expression, purification and crystallization. We prepared two EpEX variants$w t$ EpEX (residues 24-265 of EpCAM) and the non-glycosylated mutant variant (EpEXA), both with an uncleavable $\mathrm{His}_{6}$-tag at the $\mathrm{C}$ terminus. Briefly, the region of EpCAM cDNA (clone ID IRAKp961G0321Q; Source BioScience imaGenes) coding for Gln24-Lys 265 was amplified by PCR. In case of EpEXA three mutations were introduced: Asn74Gln, Asn111Gln and Asn198Gln. Next, 5' - and $3^{\prime}$-ends of the DNA fragment were extended by PCR to introduce the $\mathrm{N}$-terminal melittin signal peptide (MKFLVNVALVFMVVYISYIYA) and a C-terminal $\mathrm{His}_{6}$-tag. The resulting fragment was cloned into pFastBacl, which was in turn used to prepare recombinant baculoviruses using the Bac-to-Bac system (Invitrogen). Expression was performed using suspension cultures of Spodoptera frugiperda Sf9 cells at multiplicity of infection of 5 . The recombinant protein was collected from the medium $36 \mathrm{~h}$ post infection by dialysis and subsequent immobilized metal affinity chromatography using $\mathrm{Ni}^{2+}$-charged HisTrap columns (GE Healthcare). The peak eluate fractions were pooled and EDTA ( $\mathrm{pH}$ 8.0) was added to a final concentration of $5 \mathrm{mM}$. Following dialysis and ion-exchange chromatography on Mono Q column (GE Healthcare), the final buffer exchange was done by size-exclusion chromatography on a Superdex 200 column (GE Healthcare) equilibrated in $20 \mathrm{mM} \mathrm{Na-HEPES} \mathrm{pH}$ $8.0,100 \mathrm{mM} \mathrm{NaCl}$. The peak fraction was pooled and concentrated using Amicon centrifugal filter unit with $10 \mathrm{kDa}$ cutoff membrane (Millipore).

EpEX was prepared using the same procedure; however, the ion-exchange chromatography step was omitted. This recombinant EpEX variant is heterogeneously glycosylated as revealed by the presence of multiple bands on SDS-polyacrylamide gel electrophoresis (SDS-PAGE) gel, all at higher $M_{r}$ than the EpEXA variant (Supplementary Fig. 1a).

For crystallization only EpEX $\Delta$ was used due to higher sample homogeneity (Supplementary Fig. 1a). EpEX $\Delta$ was crystallized by sitting drop vapour diffusion at $295 \mathrm{~K}$. Here, $2 \mu \mathrm{l}$ of protein solution $\left(10 \mathrm{mg} \mathrm{ml}^{-1}\right)$ was mixed with $2 \mu \mathrm{l}$ of crystallization buffer and $0.4 \mu \mathrm{l}$ of $18 \mathrm{mM}$ n-decyl- $\beta$-D-maltopyranoside. The crystallization buffer consisted of $0.1 \mathrm{M}$ Tris- $\mathrm{HCl}$ ( $\mathrm{pH} 8.5$ ), $0.2 \mathrm{M}$ magnesium chloride and $30 \% \mathrm{w} / \mathrm{v}$ PEG 4000 . Crystals appeared within 25 days and reached dimensions $0.2 \mathrm{~mm} \times 0.3 \mathrm{~mm} \times 0.4 \mathrm{~mm}$ (Supplementary Fig. $1 \mathrm{~b}$ ).

X-ray data collection and processing. EpEX $\Delta$ crystals were directly flash-frozen in a stream of cool nitrogen $(100 \mathrm{~K})$. Screening and data collection were performed on an in-house Bruker AXS Microstar rotating copper anode X-ray generator (wavelength of $1.542 \AA$ ) equipped with Platinum 135 CCD detector. Images were processed using the PROTEUM2 Software Suite (Bruker AXS Inc.). The statistics for crystallographic data collection are presented in Table 1.

Structure determination and refinement. The structure of EpEX $\Delta$ was determined by single-wavelength anomalous dispersion by intrinsic sulphur atoms (S$\mathrm{SAD}$ ) using highly redundant data set collected from a single crystal. The same data set was used in refinement. Initial data analysis was done in XTRIAGE ${ }^{54}$ and showed the presence of one polypeptide chain per asymmetric unit. Positions of anomalous scatterers and initial phases were determined by HySS ${ }^{55}$. Here, the positions of 14 of the total 16 intrinsic sulphur atoms were resolved (12 sulphur atoms of the 6 disulphide bridges and 2 sulphur atoms of Met69 and Met73) while the sulphur atoms of surface-exposed Met231 and Met261 could not be resolved 
due to side chain disorder (Supplementary Fig. 1c). The initial model was built using AutoBuild ${ }^{56}$ implemented in PHENIX ${ }^{54}$. The complete model was built with Arp/wArp ${ }^{57-59}$. The N-terminal residue (Gln in expressed construct) was modelled as pyroGlu based on the shape of electron density blob and previous reports ${ }^{21}$. The N-terminal Gln residue observed previously indicates that small amount of expressed protein has an unmodified $\mathrm{N}$-terminal Gln residue resulting in QEExVxE N-terminal amino-acid sequence of purified EpEX $\Delta^{28}$. A large extended blob in the positive difference electron density map was identified as a crystallization additive $n$-decyl- $\beta$-D-maltopyranoside based on the shape of the blob and composition of the crystallization drop. The final model was produced after rounds of manual building in COOT $^{60}$ and refinement in PHENIX ${ }^{54}$. The quality of the final model was assessed by using MolProbity ${ }^{61}$. The Ramachandran plot distribution analysis revealed that $95.4 \%$ of residues are in the favored region and $4.6 \%$ in allowed region with no outliers. Refinement statistics are summarized in Table 1.

Structure analysis. Protein structures with similar folds were identified using the protein structure comparison service PDBeFold at the European Bioinformatics Institute $^{23}$. The model of glycosylated EpEX was prepared using GlyProt ${ }^{62}$. In this model, high-mannose chains were attached to the three asparagine residues known to be involved in otherwise heterogeneous $w t$ glycosylation ${ }^{30}$ to get a visual insight into the glycosylated molecule. Secondary structure assignments used in structure figures were performed using STRIDE ${ }^{63}$. All structure figures were prepared in PyMOL (Schroedinger) except those in Fig. $4 \mathrm{~b}$ and Supplementary Fig. 3b that were prepared in $\mathrm{VMD}^{64}$. Amino-acid sequence alignment was prepared using Clustal $\mathrm{W}^{65}$. Sequence identity as inferred from the amino-acid sequence alignment was mapped to EpEX $\Delta$ structure using the MultiSeq 2.0 plugin $^{66}$.

MD simulation. The EpCAM TM part with amino-acid sequence $\mathrm{A}^{266} \mathrm{GVIA}$ VIVVVVIAVVAGIVVLVI ${ }^{288}$ was modelled as a canonical $\alpha$-helix with acetyl $\mathrm{N}$-terminal and $\mathrm{N}$-methylamide C-terminal caps. Two such helices were embedded in 1-palmitoyl-2-oleoylphosphatidylcholine lipid bilayer patch of dimensions $100 \AA \times 100 \AA$. Helical axes were oriented perpendicularly to bilayer plain and the interhelical distance was set to $50 \AA$. This all-atom system was parameterized to a CG system using MARTINI force field and solvated ${ }^{67}$. Here, small groups of atoms (for example, amino-acid side chains) are treated as single particles allowing calculation of longer time scales. CG MD simulations were performed in NAMD 2.9 (ref. 68) using periodic boundary conditions and $10 \mathrm{fs}$ time step under constant temperature $(310 \mathrm{~K})$ and pressure $(1.01325 \mathrm{bar})$ controlled using Langevin dynamics. First, the protein part of the system was fixed and the lipids were allowed to equilibrate for $1.25 \mathrm{~ns}$ with stepwise harmonic restraints scaling. Next, the equilibrated system was used as a starting point for six independent 2 - $\mu$ s simulations using the same parameters. Obtained trajectories were analysed and visualized in $\mathrm{VMD}^{64}$ and Gromacs ${ }^{69}$. The interhelical distance was defined as the distance between centres of the two helices. The interhelical crossing angle was defined as the angle between vectors describing the two helices; vector of each helix was drawn between centres of two groups of backbone atoms (Ala270-Val273 and Ala281-Val284). To right-handed orientation, a negative value vas assigned.

Proteolytic cleavage. EpEX and EpEX $\Delta$ were incubated with human recombinant cathepsins L, S, K and B. For each enzyme, a HEPES buffer with $\mathrm{pH} 7.2$ and an additional low-pH buffer were used (sodium acetate $\mathrm{pH} 5.5$ or Bis-Tris $\mathrm{pH}$ 6.0). All buffers were $0.1 \mathrm{M}$ and contained $2 \mathrm{mM}$ EDTA and $2 \mathrm{mM}$ DTT. Cathepsin/ $\operatorname{EpEX}(\Delta)$ molar ratio was $10^{-2}$ or $10^{-3}$. Reaction mixtures were incubated at $37^{\circ} \mathrm{C}$ for $1 \mathrm{~h}$. Reactions were stopped by adding reducing SDS-PAGE sample buffer followed by boiling. Fragments were visualized on 15\% SDS-PAGE by Coomassie staining. Cleavage site was determined by $\mathrm{N}$-terminal amino-acid sequencing (Edman degradation) of electrophoretically separated fragments transferred to a polyvinylidene difluoride membrane.

Chemical crosslinking. Crosslinking of intact or trypsin-cleaved EpEX $\Delta$ was performed in PBS by treating the protein with $1.3 \mathrm{mM} \mathrm{BS} 3$ (Pierce) at $21^{\circ} \mathrm{C}$ for $30 \mathrm{~min}$. BS ${ }^{3}$ has a spacer length of $11.4 \AA$. The reaction was quenched by adding Tris buffer ( $\mathrm{pH}$ 7.4) to a final concentration of $50 \mathrm{mM}$ followed by $30 \mathrm{~min}$ incubation at $21{ }^{\circ} \mathrm{C}$. Reaction mixtures were analysed on 15\% SDS-PAGE under nonreducing and reducing conditions. Protein bands were visualized by Coomassie staining.

Docking. Two EpEX $\Delta$ cis-dimers (chain labels A and B in one dimer and C and D in second dimer) were used in docking experiments to construct a model of the trans-tetrameric unit. Calculations were performed using HADDOCK running on WeNMR grid $^{70}$. The N-terminal pyroGlu residue was changed to Gln because modified residues are not generally accepted in docking simulations. Histidine protonation states and flexible segments were defined automatically and centre of mass restraint was used to enforce contact between the molecules. $\mathrm{C} 2$ point symmetry restraint was imposed between the two dimers (AB-CD). An additional calculation was performed where $\mathrm{C} 2$ point symmetry restrains between subunits in each subunit pair $(\mathrm{A}-\mathrm{C}, \mathrm{A}-\mathrm{D}, \mathrm{B}-\mathrm{C}, \mathrm{B}-\mathrm{D})$ were used to limit calculation results to tetrameric units with perpendicular C2 rotation axes. Docking solutions were first ranked according to their HADDOCK and $Z$-scores, the latter indicating separation of a cluster of solutions from the average in terms of score (better solutions have more negative $Z$-scores), and further ranked according to the buried surface area and contacts between ND. Both docking runs yielded similar results. In the models from the best docking cluster $(Z=-1.2)$, the relative angle between the two cis-dimers was $30^{\circ}$, while in the models from second $(Z=-0.9)$ and third best clusters $(Z=0.1)$, the relative angle was $150^{\circ}$ (ND's already in contact) and $75^{\circ}$, respectively, with a smaller buried surface area. For figure preparation, the best model from additional symmetry-restrained docking run was used.

\section{References}

1. Herlyn, D. et al. Efficient selection of human tumor growth-inhibiting monoclonal antibodies. J. Immunol. Methods 73, 157-167 (1984).

2. Went, P. et al. Frequent high-level expression of the immunotherapeutic target Ep-CAM in colon, stomach, prostate and lung cancers. Br. J. Cancer 94, 128-135 (2006).

3. Sundberg, M. et al. CD marker expression profiles of human embryonic stem cells and their neural derivatives, determined using flow-cytometric analysis, reveal a novel CD marker for exclusion of pluripotent stem cells. Stem Cell Res. 2, 113-124 (2009).

4. Ng, V. Y., Ang, S. N., Chan, J. X. \& Choo, A. B. H. Characterization of epithelial cell adhesion molecule as a surface marker on undifferentiated human embryonic stem cells. Stem Cells 28, 29-35 (2010).

5. Simon, M., Stefan, N., Plückthun, A. \& Zangemeister-Wittke, U. Epithelial cell adhesion molecule-targeted drug delivery for cancer therapy. Expert Opin. Drug Deliv. 10, 451-468 (2013).

6. Ruf, P. et al. Pharmacokinetics, immunogenicity and bioactivity of the therapeutic antibody catumaxomab intraperitoneally administered to cancer patients. Br. J. Clin. Pharmacol. 69, 617-625 (2010).

7. Schnell, U. et al. Absence of cell-surface EpCAM in congenital tufting enteropathy. Hum. Mol. Genet 22, 2566-2571 (2013).

8. Litvinov, S. V., Velders, M. P., Bakker, H. A., Fleuren, G. J. \& Warnaar, S. O. Ep-CAM: a human epithelial antigen is a homophilic cell-cell adhesion molecule. J. Cell Biol. 125, 437-446 (1994).

9. Litvinov, S. V., Bakker, H. A., Gourevitch, M. M., Velders, M. P. \& Warnaar, S. O. Evidence for a role of the epithelial glycoprotein 40 (Ep-CAM) in epithelial cell-cell adhesion. Cell Adhes. Commun. 2, 417-428 (1994).

10. Balzar, M. et al. The structural analysis of adhesions mediated by Ep-CAM. Exp. Cell Res. 246, 108-121 (1999).

11. Trebak, M. et al. Oligomeric state of the colon carcinoma-associated glycoprotein GA733-2 (Ep-CAM/EGP40) and its role in GA733-mediated homotypic cell-cell adhesion. J. Biol. Chem. 276, 2299-2309 (2001).

12. Balzar, M. et al. Epidermal growth factor-like repeats mediate lateral and reciprocal interactions of Ep-CAM molecules in homophilic adhesions. Mol. Cell. Biol. 21, 2570-2580 (2001).

13. Balzar, M. et al. Cytoplasmic tail regulates the intercellular adhesion function of the epithelial cell adhesion molecule. Mol. Cell. Biol. 18, 4833-4843 (1998).

14. Litvinov, S. V. et al. Epithelial cell adhesion molecule (Ep-CAM) modulates cell-cell interactions mediated by classic cadherins. J. Cell Biol. 139, 1337-1348 (1997).

15. Winter, M. J. et al. Expression of Ep-CAM shifts the state of cadherin-mediated adhesions from strong to weak. Exp. Cell Res. 285, 50-58 (2003).

16. Ladwein, M. et al. The cell-cell adhesion molecule EpCAM interacts directly with the tight junction protein claudin-7. Exp. Cell Res. 309, 345-357 (2005).

17. Lei, Z. et al. EpCAM contributes to formation of functional tight junction in the intestinal epithelium by recruiting claudin proteins. Dev. Biol. 371, 136-145 (2012)

18. Wu, C.-J., Mannan, P., Lu, M. \& Udey, M. C. Epithelial cell adhesion molecule (EpCAM) regulates claudin dynamics and tight junctions. J. Biol. Chem. 288, 12253-12268 (2013).

19. Maetzel, D. et al. Nuclear signalling by tumour-associated antigen EpCAM. Nat. Cell Biol. 11, 162-171 (2009).

20. Denzel, S. et al. Initial activation of EpCAM cleavage via cell-to-cell contact. BMC Cancer 9, 402 (2009).

21. Chong, J. M. \& Speicher, D. W. Determination of disulphide bond assignments and $\mathrm{N}$-glycosylation sites of the human gastrointestinal carcinoma antigen GA733-2 (CO17-1A, EGP, KS1-4, KSA, and Ep-CAM). J. Biol. Chem. 276, 5804-5813 (2001).

22. Calvanese, L. et al. Solution structure of mouse Cripto CFC domain and its inactive variant Trp107Ala. J. Med. Chem. 49, 7054-7062 (2006).

23. Krissinel, E. \& Henrick, K. Secondary-structure matching (SSM), a new tool for fast protein structure alignment in three dimensions. Acta Crystallogr. D Biol. Crystallogr. 60, 2256-2268 (2004).

24. Salah, Z., Alian, A. \& Aqeilan, R. I. WW domain-containing proteins: retrospectives and the future. Front. Biosci. 17, 331-348 (2012).

25. Molina, F., Bouanani, M., Pau, B. \& Granier, C. Characterization of the type-1 repeat from thyroglobulin, a cysteine-rich module found in proteins from different families. Eur. J. Biochem. 240, 125-133 (1996). 
26. Hardin, J. W., Hu, Y. X. \& McKay, D. B. Structure of the RNA binding domain of a DEAD-box helicase bound to its ribosomal RNA target reveals a novel mode of recognition by an RNA recognition motif. J. Mol. Biol. 402, 412-427 (2010).

27. Primo, M. E. et al. Structure of the mature ectodomain of the human receptortype protein-tyrosine phosphatase IA-2. J. Biol. Chem. 283, 4674-4681 (2008).

28. Pavšič, M. \& Lenarčič, B. Expression, crystallization and preliminary X-ray characterization of the human epithelial cell-adhesion molecule ectodomain. Acta Crystallogr. Sect. F Struct. Biol. Cryst. Commun. 67, 1363-1366 (2011).

29. Krissinel, E. \& Henrick, K. Inference of macromolecular assemblies from crystalline state. J. Mol. Biol. 372, 774-797 (2007).

30. Münz, M., Fellinger, K., Hofmann, T., Schmitt, B. \& Gires, O. Glycosylation is crucial for stability of tumour and cancer stem cell antigen EpCAM. Front. Biosci. 13, 5195-5201 (2008).

31. Fernsten, P. D., Pekny, K. W., Reisfeld, R. A. \& Walker, L. E. Biosynthesis and glycosylation of the carcinoma-associated antigen recognized by monoclonal antibody KS1/4. Cancer Res. 50, 4656-4663 (1990).

32. Thampoe, I. J., Ng, J. S. \& Lloyd, K. O. Biochemical analysis of a human epithelial surface antigen: differential cell expression and processing. Arch. Biochem. Biophys. 267, 342-352 (1988).

33. Perez, M. S. \& Walker, L. E. Isolation and characterization of a cDNA encoding the KS1/4 epithelial carcinoma marker. J. Immunol. 142, 3662-3667 (1989).

34. Schön, M. P. et al. Biochemical and immunological characterization of the human carcinoma-associated antigen MH 99/KS 1/4. Int. J. Cancer 55, 988-995 (1993).

35. Psachoulia, E., Fowler, P. W., Bond, P. J. \& Sansom, M. S. P. Helix-helix interactions in membrane proteins: coarse-grained simulations of glycophorin a helix dimerization. Biochemistry 47, 10503-10512 (2008).

36. Bevec, T., Stoka, V., Pungerčič, G., Dolenc, I. \& Turk, V. Major histocompatibility complex class II-associated p41 invariant chain fragment is a strong inhibitor of lysosomal cathepsin L. J. Exp. Med. 183, 1331-1338 (1996).

37. Meh, P., Pavšič, M., Turk, V., Baici, A. \& Lenarčič, B. Dual concentrationdependent activity of thyroglobulin type-1 domain of testican: specific inhibitor and substrate of cathepsin L. Biol. Chem. 386, 75-83 (2005).

38. Sitar, T., Popowicz, G. M., Siwanowicz, I., Huber, R. \& Holak, T. A. Structural basis for the inhibition of insulin-like growth factors by insulin-like growth factor-binding proteins. Proc. Natl Acad. Sci. USA 103, 13028-13033 (2006).

39. Novinec, M., Kordišs, D., Turk, V. \& Lenarčič, B. Diversity and evolution of the thyroglobulin type-1 domain superfamily. Mol. Biol. Evol. 23, 744-755 (2006).

40. Kuhn, S. et al. A Complex of EpCAM, Claudin-7, CD44 variant isoforms, and tetraspanins promotes colorectal cancer progression. Mol. Cancer Res. 5, 553-567 (2007).

41. van Sluis, R. et al. In vivo imaging of extracellular $\mathrm{pH}$ using $1 \mathrm{H}$ MRSI. Magn. Reson. Med. 41, 743-750 (1999).

42. Gocheva, V. \& Joyce, J. A. Cysteine cathepsins and the cutting edge of cancer invasion. Cell Cycle 6, 60-64 (2007).

43. Pauli, C. et al. Tumor-specific glycosylation of the carcinoma-associated epithelial cell adhesion molecule EpCAM in head and neck carcinomas. Cancer Lett. 193, 25-32 (2003).

44. Casasnovas, J. M., Springer, T. A., Liu, J. H., Harrison, S. C. \& Wang, J. H. Crystal structure of ICAM-2 reveals a distinctive integrin recognition surface. Nature 387, 312-315 (1997).

45. Boggon, T. J. et al. C-cadherin ectodomain structure and implications for cell adhesion mechanisms. Science 296, 1308-1313 (2002).

46. Bryan, R. T. et al. Urinary EpCAM in urothelial bladder cancer patients: characterisation and evaluation of biomarker potential. Br. J. Cancer 110, 679-685 (2014)

47. Hachmeister, M. et al. Regulated intramembrane proteolysis and degradation of murine epithelial cell adhesion molecule mEpCAM. PLOS ONE 8, e71836 (2013).

48. Shimizu, H. et al. Crystal structure of an active form of BACE1, an enzyme responsible for amyloid beta protein production. Mol. Cell. Biol. 28, 3663-3671 (2008).

49. Schnell, U., Kuipers, J. \& Giepmans, B. N. G. EpCAM proteolysis: new fragments with distinct functions? Biosci. Rep. 33, e00030 (2013).

50. Sivagnanam, M. et al. Identification of EpCAM as the gene for congenital tufting enteropathy. Gastroenterology 135, 429-437 (2008).

51. Goldstein, A. S. et al. Trop2 identifies a subpopulation of murine and human prostate basal cells with stem cell characteristics. Proc. Natl Acad. Sci. U.S.A. 105, 20882-20887 (2008).

52. Cubas, R., Li, M., Chen, C. \& Yao, Q. Trop2: a possible therapeutic target for late stage epithelial carcinomas. Biochim. Biophys. Acta 1796, 309-314 (2009).

53. Stoyanova, T. et al. Regulated proteolysis of Trop2 drives epithelial hyperplasia and stem cell self-renewal via $\beta$-catenin signaling. Genes Dev. 26, 2271-2285 (2012).

54. Adams, P. D. et al. PHENIX: a comprehensive Python-based system for macromolecular structure solution. Acta Crystallogr. D Biol. Crystallogr 66, 213-221 (2010)
55. Grosse-Kunstleve, R. W. \& Adams, P. D. Substructure search procedures for macromolecular structures. Acta Crystallogr. D Biol. Crystallogr 59, 1966-1973 (2003).

56. Terwilliger, T. C. et al. Iterative model building, structure refinement and density modification with the PHENIX AutoBuild wizard. Acta Crystallogr. D Biol. Crystallogr 64, 61-69 (2008).

57. Perrakis, A., Morris, R. \& Lamzin, V. S. Automated protein model building combined with iterative structure refinement. Nat. Struct. Biol. 6, 458-463 (1999).

58. Mooij, W. T. M., Cohen, S. X., Joosten, K., Murshudov, G. N. \& Perrakis, A. 'Conditional Restraints': restraining the free atoms in ARP/wARP. Structure. 17, 183-189 (2009)

59. Murshudov, G. N., Vagin, A. A. \& Dodson, E. J. Refinement of macromolecular structures by the maximum-likelihood method. Acta Crystallogr. D Biol. Crystallogr. 53, 240-255 (1997).

60. Emsley, P. \& Cowtan, K. Coot: model-building tools for molecular graphics. Acta Crystallogr. D Biol. Crystallogr. 60, 2126-2132 (2004).

61. Chen, V. B. et al. MolProbity: all-atom structure validation for macromolecular crystallography. Acta Crystallogr. D Biol. Crystallogr. 66, 12-21 (2010).

62. Bohne-Lang, A. \& Lieth, von der, C.-W. GlyProt: in silico glycosylation of proteins. Nucleic Acids Res. 33, W214-W219 (2005).

63. Frishman, D. \& Argos, P. Knowledge-based protein secondary structure assignment. Proteins 23, 566-579 (1995).

64. Humphrey, W., Dalke, A. \& Schulten, K. VMD: visual molecular dynamics. J. Mol. Graph. 14, 33-38-27-8 (1996)

65. Larkin, M. A. et al. Clustal W and Clustal X version 2.0. Bioinformatics. 23, 2947-2948 (2007).

66. Roberts, E., Eargle, J., Wright, D. \& Luthey-Schulten, Z. MultiSeq: unifying sequence and structure data for evolutionary analysis. BMC Bioinformatics 7 , 382 (2006).

67. Marrink, S. J., Risselada, H. J., Yefimov, S., Tieleman, D. P. \& de Vries, A. H. The MARTINI force field: coarse grained model for biomolecular simulations. J. Phys. Chem. B 111, 7812-7824 (2007).

68. Phillips, J. C. et al. Scalable molecular dynamics with NAMD. J. Comput. Chem. 26, 1781-1802 (2005).

69. Hess, B., Kutzner, C., van der Spoel, D. \& Lindahl, E. GROMACS 4: algorithms for highly efficient, load-balanced, and scalable molecular simulation. J. Chem. Theory Comput. 4, 435-447 (2008).

70. de Vries, S. J., van Dijk, M. \& Bonvin, A.M.J.J. The HADDOCK web server for data-driven biomolecular docking. Nat. Protoc. 5, 883-897 (2010).

\section{Acknowledgements}

We thank B. Sjöblom for help during X-ray diffraction data collection at Max F. Perutz Laboratories, University of Vienna. We are grateful to M. Novinec for samples of recombinant human cathepsins, and to Prof. S. Polanc for help with DMU identification (both from Department of Chemistry and Biochemistry, Faculty of Chemistry and Chemical Technology, University of Ljubljana). We thank Prof. A. Baici for helpful discussion. The software packages VMD (http://www.ks.uiuc.edu/Research/vmd/) and NAMD (http://www.ks.uiuc.edu/Research/namd/) were developed by the Theoretical and Computational Biophysics Group in the Beckman Institute for Advanced Science and Technology at the University of Illinois at Urbana-Champaign. The WeNMR project (European FP7 e-Infrastructure grant, contract no. 261572, www.wenmr.eu), supported by the national GRID Initiatives of Belgium, France, Italy, Germany, the Netherlands (via the Dutch BiG Grid project), Portugal, Spain, UK, South Africa, Taiwan and the Latin America GRID infrastructure via the Gisela project is acknowledged for the use of web portals, computing and storage facilities. This work was supported by the Slovenian Research Agency (grant J1-2017).

\section{Author contributions}

M.P. and B.L. designed the project. M.P. performed all of the experiments. K.D.-C devised phasing strategy. M.P. and G.G. performed final structure refinement. M.P. and B.L. analysed the results and wrote the manuscript.

\section{Additional information}

Accession codes: Coordinates and structural factors have been deposited in the Protein Data Bank under accession code $4 \mathrm{MZV}$.

Supplementary Information accompanies this paper at http://www.nature.com/ naturecommunications

Competing financial interests: The authors declare no competing financial interests.

Reprints and permissions information is available online at http://npg.nature.com/ reprintsandpermissions

How to cite this article: Pavšič, M. et al. Crystal structure and its bearing towards an understanding of key biological functions of EpCAM. Nat. Commun. 5:4764 doi: $10.1038 / 5764$ (2014). 